\title{
Time-Resolved Reflectivity and Temperature Measurements During Laser Irradiation of Crystalline Silicon
}

\author{
Michael Diez*, Mawuli Ametowobla** and Thomas Graf ${ }^{* * *}$ \\ ${ }^{*}$ Robert Bosch GmbH, C/CEP, P.O. Box 300220, 70442 Stuttgart, Germany \\ E-mail: Michael.Diez@de.bosch.com \\ ** Robert Bosch GmbH, CR/APJ2, Robert-Bosch-Campus 1, 71272 Renningen, Germany \\ *** Institut für Strahlwerkzeuge, University Stuttgart, Pfaffenwaldring 43, 70569 Stuttgart, Germany
}

\begin{abstract}
Time-resolved reflectivity measurements have been used to characterize the temperaturedependence of the reflectivity of crystalline silicon between room temperature and the melting point during pulsed laser irradiation ( $\lambda=532 \mathrm{~nm}$, pulse durations between $50 \mathrm{~ns}$ and $600 \mathrm{~ns})$. Based on these measurements, the reflectivity $R_{\mathrm{Si}}\left(T_{\mathrm{m}}\right)$ of the hot solid just before the onset of melting was determined for the probe wavelength of $\lambda=632.8 \mathrm{~nm}$. For this wavelength, $R_{\mathrm{Si}}\left(T_{\mathrm{m}}\right)$ was found to be $39.8 \% \pm 0.6 \%$. The increase of the measured reflectivity was correlated to the laser-induced temperature increase of the silicon surface as calculated by solving the $1 \mathrm{D}$ heat-conduction equation. This approach allows to indirectly determine the temperature in the laser-material interaction zone on the surface of a silicon sample during pulsed laser irradiation with sub-nanosecond time resolution by monitoring its reflectivity.
\end{abstract}

DOI: 10.2961/jlmn.2017.03.0010

Keywords: reflectivity, silicon, laser, pump-probe, temperature dependence, melting point

\section{Introduction}

Processing of silicon by using laser radiation is becoming more and more important in the photovoltaic and semiconductor industry. Annealing, structuring and ablation processes based on short and ultra-short [1] pulsed lasers start to compete with established manufacturing processes [2]. Thus, the precise knowledge of the interaction mechanisms between the laser radiation and the semiconductor is of major importance, especially for applications with a narrow processing window, e. g. processes that are tailored to run close to the melting or the ablation threshold. The optical properties (reflectivity and absorption) are of special interest since they determine the amount of pulse energy or intensity that can be coupled into the semiconductor to drive the laser process. Although a lot of experimental data is available for the temperature dependence of the reflectivity of crystalline silicon $R_{\mathrm{Si}}$, these data almost solely provide information on the reflectivity at discrete temperature values $T$ which are either far below the melting temperature [3 - 10] or refer to the liquid state [11, 12]. A consistent experimental description of $R_{\mathrm{Si}}(T)$ in the entire temperature range between room temperature $\left(T_{\mathrm{RT}}=\right.$ $300 \mathrm{~K})$ and melting temperature $\left(T_{\mathrm{m}}=1683 \mathrm{~K}\right)$ is still missing. This is mainly due to the formation of oxide layers during the time between heating and the measurement of $R_{\mathrm{Si}}(T)$ e.g. by means of ellipsometry, which makes it difficult to determine accurate data at elevated temperatures [5].

In this work, the evolution of the reflectivity of crystalline silicon is consistently characterized in the entire range between $T_{\mathrm{RT}}$ and $T_{\mathrm{m}}$ by means of time-resolved measurements. In order to cover the whole temperature range, a pump-probe setup with a nanosecond laser as the heating source was chosen. Since the time scale for the whole heating and measuring procedure is only a few hundred nanoseconds, oxidation can be assumed to be negligible $[13,14]$.

\section{Experimental Details}

The experimental pump-probe setup used for the timeresolved measurements of the reflectivity is schematically illustrated in Fig. 1.

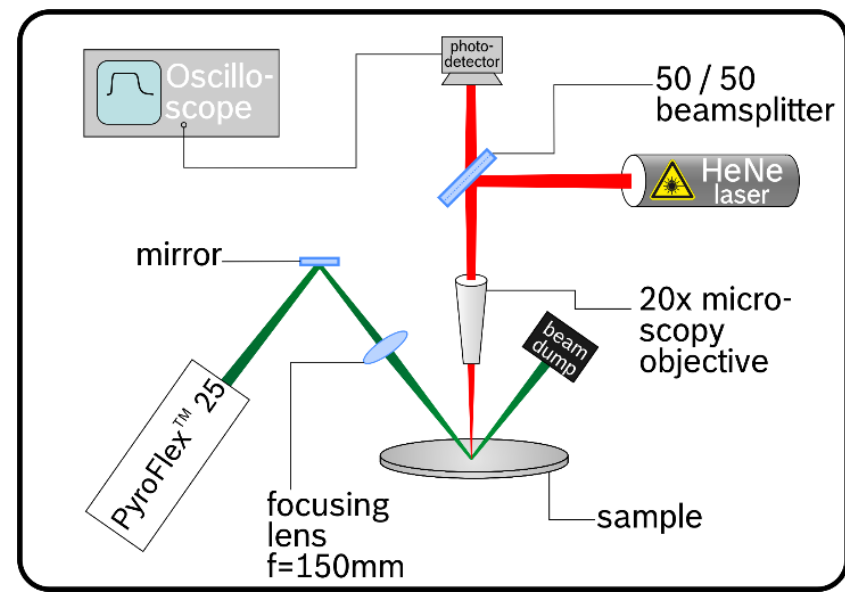

Fig. 1 Setup for time-resolved reflectivity measurements. The probe beam from the HeNe laser is focused on the sample to a diameter of $D_{\text {probe }}<25 \mu \mathrm{m}$. The pump beam of the PyroFlex ${ }^{\mathrm{TM}}$ laser is aligned to the same location but with a significantly larger diameter. The reflected probe beam is detected by an avalanche photodiode whose signals are recorded by a fast oscilloscope. 
The setup basically consisted of a pump laser to irradiate the surface of the samples, a cw HeNe probe laser to assess the changes of the reflectivity of the heated surface and an avalanche photodiode connected to a fast oscilloscope to monitor the changes. The reflectivity experiments were conducted on optically polished (100) $n$-Type Si wafers with a specific resistance of $\rho=4 \pm 1 \Omega \mathrm{cm}$. The pump laser was a pulsed PyroFlex ${ }^{\mathrm{TM}}$ 25-GR fiber laser operating at a wavelength of $532 \mathrm{~nm}$ and with $\mathrm{M}^{2}<1.3$. Due to the special architecture of the laser source, it was possible to adjust the pulse duration in the range from approximately two nanoseconds to several 100s of nanoseconds with negligible influence on the other laser parameters. This feature enabled us to investigate the effect of different pulse durations in one experimental setup, avoiding any experimental influences resulting from different laser sources or optical setups. The possibility to adjust the pulse duration and the fact that the temporal intensity profile of a single laser pulse was virtually rectangular - while being almost Gaussian in space - allowed us to precisely control the irradiation conditions (pulse intensity and duration). The intensity of the pump beam was adjusted by a half-wave plate and a polarizer. The beam was focused with a bi-convex lens with a focal length of $f=150 \mathrm{~mm}$. The angle of incidence was adjusted to be circa $30^{\circ}$ (to the surface's normal). This resulted in an elliptical spot on the sample surface of $>100 \mu \mathrm{m}$ by $>150 \mu \mathrm{m}$ microns, depending on the pulse duration used. It was anticipated that any native oxide layer that is present on the sample surface would not interfere with the measurements, since the penetration depth of the probe beam would by far exceed the native oxide thickness (1 $3 \mathrm{~nm}$ ) [15]. Thus, a simple cleaning of the surface by isopropyl alcohol was sufficient to remove organic contamination prior to the irradiation in ambient environment.

The probe beam was supplied by a single mode $4 \mathrm{~mW}$ cw HeNe laser emitting at a wavelength of $\lambda=632.8 \mathrm{~nm}$. The beam was focused by a $20 \mathrm{x}$ microscope objective to a diameter of $D_{\text {probe }}<25 \mu \mathrm{m}$, resulting in a spot area that was more than one order of magnitude smaller than the spot area of the pump beam. This difference in spot size on the sample surface made sure that the investigated laser-material interaction area was irradiated homogeneously by the PyroFlex $^{\mathrm{TM}}$ 25-GR laser beam. The part of the probe beam's intensity that was reflected by the sample surface passed a $50 / 50$ beam splitter and was detected by an avalanche photodiode with a bandwidth of $50 \mathrm{MHz}$. To ensure a homogeneous illumination of the active detection area of the photodiode $\left(0.79 \mathrm{~mm}^{2}\right)$, a diffuser plate was mounted between the optical bandpass filter $(632.8 \mathrm{~nm} \pm 2 \mathrm{~nm})$ and the detection area. The responsitivity of the photodiode for $\lambda=632.8 \mathrm{~nm}$ was $20 \mathrm{~mA} / \mathrm{W}$. The voltage signals were recorded by a fast oscilloscope with $20 \mathrm{GS} / \mathrm{s}$.

\section{Results and Discussion}

Fig. 2 shows the voltage signal of the photodiode (left ordinate) during irradiation with a laser pulse with a duration of $\tau_{\mathrm{p}}=300 \mathrm{~ns}$ and an intensity well above the melting threshold. This signal was converted into the time-resolved evolution of the reflectivity of crystalline silicon (right ordinate) for $\lambda=632.8 \mathrm{~nm}$ with the help of equation (2) as explained below. The normalized temporal intensity profile of the pump pulse is shown in the small insert on the right hand side of Fig. 2.

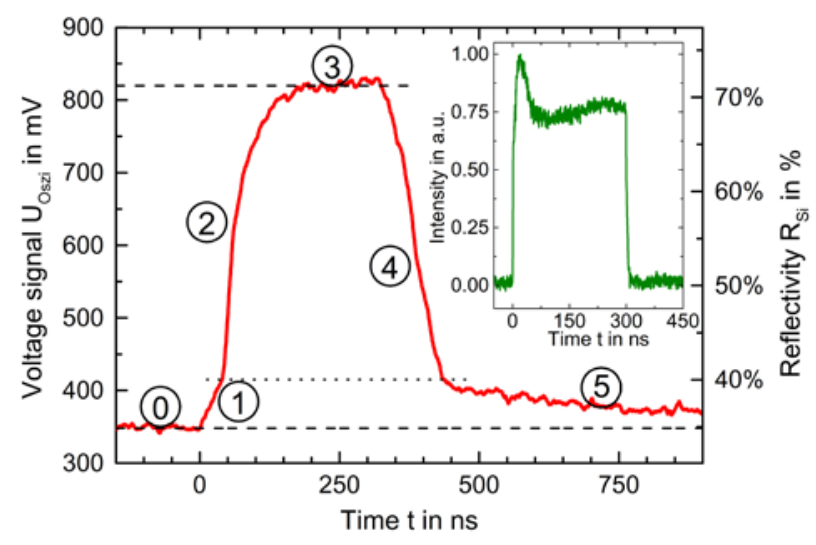

Fig. 2 Voltage signal (left ordinate) and evolution of the reflectivity of c-Si (right ordinate) upon laser irradiation by a pulse with a duration of $\tau_{\mathrm{p}}=300 \mathrm{~ns}$. The intensity of the pump pulse was set to be high enough to melt the surface. The temporal evolution of the pulse is shown by the insert on the right. The dashed lines are guides to the eye that mark the characteristic sections and kinks in the $U_{\text {oszi }}(t)$-signal, see text.

\subsection{Relation between voltage signal and reflectivity of the probed surface}

The voltage signal $U_{\text {Oszi }}(t)$ generated by the avalanche photodiode increases linearly with the reflected intensity $I_{\text {refl }}$ of the probe beam. $I_{\text {refl }}$ is in turn proportional to the temperature dependent reflectivity of the silicon surface so that

$U_{\mathrm{Oszi}}=A \cdot I_{\mathrm{refl}}+B=A \cdot R_{S i}(T(t)) \cdot I_{\mathrm{HeNe}}+B$,

where $A$ is a measure for the responsitivity of the photodiode and $B$ stands for the offset of the signal. $I_{\mathrm{HeNe}}$ is the intensity emitted by the probe laser. Before the pump pulse irradiates the sample ( $\mathrm{t}<0 \mathrm{~ns}$ ), a constant voltage signal is recorded $\left(U_{0}=348 \pm 5 \mathrm{mV}\right.$, marked as (0) in Fig. 2). This voltage level corresponds to the reflectivity of crystalline silicon at $\lambda=$ $632.8 \mathrm{~nm}$ and at room temperature which is known to be $R_{\mathrm{Si}}\left(T_{\mathrm{RT}}\right)=34.8 \%$ [3]. After approximately 150 ns of laser irradiation, the voltage level reached an almost constant level again ( $U_{1}=820 \pm 2.5 \mathrm{mV}$, marked as (3) in Fig. 2). This value corresponds to the reflectivity of liquid silicon $[4,13$, 14] which is $R_{\mathrm{Si}}\left(T_{\text {liq }}\right)=71.2 \%$ [14] for $\lambda=632.8 \mathrm{~nm}$. Inserting these values for $R_{\mathrm{Si}}\left(T_{\mathrm{RT}}\right)$ and $R_{\mathrm{Si}}\left(T_{\text {liq }}\right)$ together with $U_{0}$ and $U_{1}$ into equation (1), one finds the values for $A=$ $1354.3 \mathrm{mV} / \mathrm{W}$ and $B=-123.5 \mathrm{mV}$. Solving equation (1) for $R_{\mathrm{Si}}(t)=R_{\mathrm{Si}}(T(t))$, where $t$ is time, then yields

$R_{S i}(t)=\frac{U_{O s z i}(t)+123.5 \mathrm{mV}}{1354.3 \frac{\mathrm{mV}}{W} \cdot I_{\mathrm{HeNe}}}$.

Approximately 25 ns after the end of the laser pulse, the voltage signal starts to decrease due to the cooling of the sample. The end of the phase transition from liquid to solid is indicated by the kink in the slope of the signal at $t \approx 435 \mathrm{~ns}$ (intersection between (4)and (5) in Fig. 2). 


\subsection{Reflectivity of solid silicon at $T_{\mathrm{m}}$}

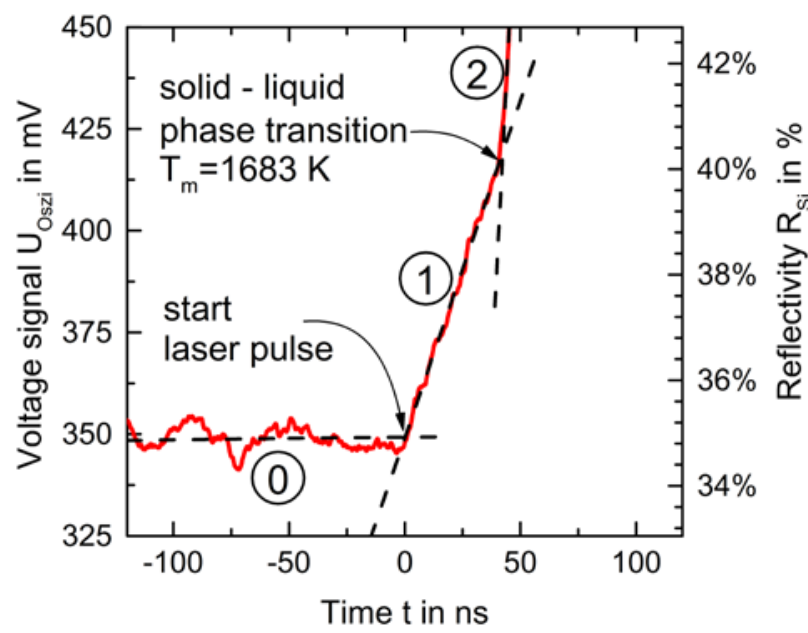

Fig. 3 Magnification of the first 100 ns of the measured voltage signal and reflectivity from Fig. 2. The dashed lines correspond to

a linear approximation of the signal during the solid phase

$(t<0 \mathrm{~ns}$, marked as (0)), the heating phase for temperatures between $T_{\mathrm{RT}}$ and $T_{\mathrm{m}}(0<\mathrm{t}<42.3 \mathrm{~ns}$, marked as (1) ) as well as the transition phase from solid to liquid ( $\mathrm{t}>42.3 \mathrm{~ns}$, marked as (2)).

Fig. 3 shows a magnification of the voltage signal and reflectivity as measured during the first $100 \mathrm{~ns}$ of irradiation by the pump beam. The dashed lines are linear approximations to the voltage pattern and help to identify the start of the laser irradiation (intersection between (1) and (1)) as well as the onset of the solid-liquid phase transition (intersection between (1) and (2)) at $T_{\mathrm{m}}=1683 \mathrm{~K}$. Hence, the reflectivity of the hot solid silicon just before the onset of melting $R_{\mathrm{Si}}\left(T_{\mathrm{m}}\right)$ can be determined for the probe wavelength $\lambda=$ $632.8 \mathrm{~nm}$ by this interpretation of the detected voltage signals.

The values of $R_{\mathrm{Si}}\left(T_{\mathrm{m}}\right)$ thus determined for different pump pulse energies and different pulse durations are shown in Fig. 4. Within the experimental resolution, it is shown that the value for $R_{\mathrm{Si}}\left(T_{\mathrm{m}}\right)$ neither depends on the pulse energy or laser fluence nor on the pulse duration. The mean value is found to be $R_{\mathrm{Si}}\left(T_{\mathrm{m}}\right)=39.8 \%$ with a standard deviation of $0.6 \%$.

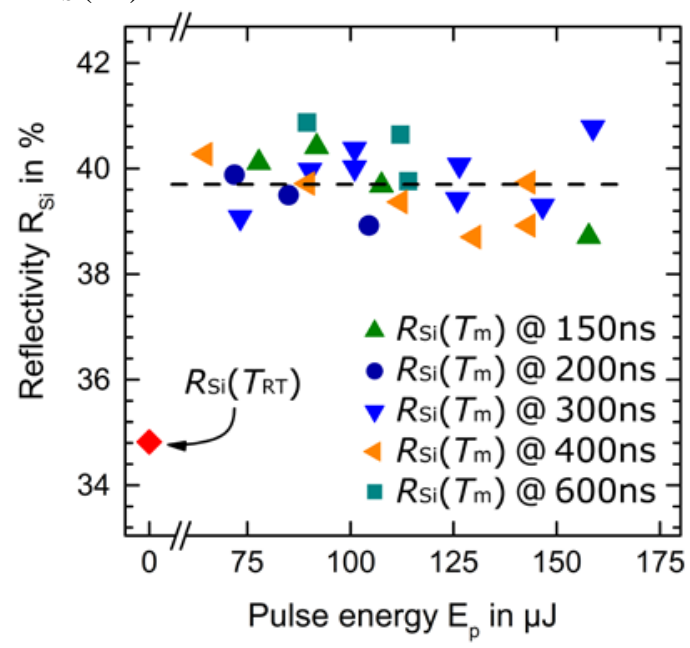

Fig. 4 Reflectivity at $\lambda=632.8 \mathrm{~nm}$ of the solid silicon at the melting point for different pulse durations as a function of pulse energy. The dashed line corresponds to the mean value of

$R_{\mathrm{Si}}\left(T_{\mathrm{m}}\right)=39.8 \%$. The reflectivity value at room temperature is shown at $E_{\mathrm{p}}=0 \mu \mathrm{J}$.

\subsection{In-situ determination of the laser-induced tempera- ture increase}

The temperature $T$ in a laser treated area can generally be calculated by solving the 3D heat-conduction equation

$\nabla k \nabla T-\frac{\partial T}{\partial t}=-\frac{1}{\kappa} \cdot q(x, y, z, t)$

where $k$ is the thermal diffusivity, $\kappa$ the thermal conductivity and $q(x, y, z, t)$ the heat source or heat sink respectively [16].

Since we are only interested in the temperature increase in the center of the laser-silicon interaction area where the intensity distribution of the laser beam perpendicular to the beam axis is homogenous and the lateral dimensions of the irradiated area are approx. two orders of magnitude larger than the thermal penetration depth $l_{\mathrm{th}}=2 \sqrt{k \cdot t}\left(l_{\mathrm{th}}<14.5 \mu \mathrm{m}\right.$ for the time scales under consideration), the reduction of the problem to only one dimension is reasonable:

$\frac{\partial}{\partial z}\left(k \frac{\partial T}{\partial z}\right)-\frac{\partial T}{\partial t}=-\frac{1}{\kappa} \cdot q(z, t)$.

In addition, Gatskevich et al. have shown that the results (e.g. values for surface temperature) of such a simplified 1D approach agree very well with the results of more sophisticated 3D simulations [17]. For example, comparing the 1D and 3D approach, the calculated values for the maximum surface temperature differ by less than $2 \%$, when the temperature dependences of the thermal diffusivity $k(T)$ and the thermal conductivity $\kappa(T)$ are considered in the calculations [17].

Furthermore, due to the low optical (ca. $1.3 \mu \mathrm{m}$ for $\lambda=532 \mathrm{~nm}$ ) and thermal penetration depths in relation to the spot area of the pump beam, the volumetric heat source $q(z, t)$ can be considered as a surface source $(z=0)$ with constant dimensions during the time of irradiation.

The solution of the 1D heat conduction equation for the present problem (a semi-infinite body irradiated by a surface heat source with a spatially and temporally constant intensity distribution during the pulse duration $\tau_{\mathrm{p}}$ ) is taken from [16]. The expression for the temperature profile in the center of the irradiated area and depth $z$

$T(z, t)=T_{R T}+\frac{I_{\mathrm{abs}}(t)}{\kappa}[\underbrace{l_{t h} \cdot \operatorname{ierfc}\left(\frac{z}{l_{t h}}\right)}_{\text {heating phase }}-\underbrace{l_{t h}^{*} \cdot \operatorname{ierfc}\left(\frac{z}{l_{t h}^{*}}\right)}_{\text {cooling phase }}]$

consists of a term for the heating phase and a term for the cooling phase $\left(t>\tau_{\mathrm{p}}\right)$, where $l_{t h}^{*}=2 \sqrt{k \cdot\left(t-\tau_{p}\right)} \cdot I_{\mathrm{abs}}(t)$ represents the intensity absorbed until time $t$ and $\operatorname{ierfc}(\cdot)$ stands for the complementary error function with $\operatorname{ierfc}(0)=1 / \sqrt{\pi}$.

To apply the 1D heat-conduction equation (5) to the following problem, the observed time span for laser-induced heating $t<\tau_{\mathrm{p}}$ is considered as a sequence of discrete elements of $t_{1} . . t_{\mathrm{n}}, n \in \mathbb{N}, t_{n} \leq \tau_{\mathrm{p}}$. For a sufficiently small distance between the elements of the sequence, the intensity absorbed until $t_{\mathrm{n}}, I_{\mathrm{abs}}\left(t_{\mathrm{n}}\right)$, is only insignificantly higher than $I_{\mathrm{abs}}\left(t_{\mathrm{n}-1}\right)$, therefore it holds that $\forall \varepsilon>0, \varepsilon \in \mathbb{R},\left|I_{\mathrm{abs}}\left(t_{\mathrm{n}}\right)-I_{\mathrm{abs}}\left(t_{\mathrm{n}-1}\right)\right|<\varepsilon$. Thus, the time-dependent temperature increase $\Delta T\left(t_{n}\right)$ of the silicon surface (i. e. $z=0$ ) after time $t_{\mathrm{n}}<\tau_{\mathrm{p}}$

$\Delta T\left(t_{\mathrm{n}}\right)=\frac{2 \cdot\left(I_{\mathrm{abs}}\left(t_{\mathrm{n}-1}\right)+\varepsilon\right)}{\kappa} \sqrt{\frac{k}{\pi} \cdot t_{\mathrm{n}}}$ 
can be expressed with the help of equation (5) as a function of the absorbed intensity $I_{\mathrm{abs}}\left(t_{\mathrm{n}-1}\right)$ (instead of $I_{\mathrm{abs}}\left(t_{\mathrm{n}}\right)$ ), the thermal conductivity $\kappa$, and the thermal diffusivity $k$.

In the present approach, $\left(t_{n}-t_{n-1}\right)$ was chosen to match the sampling rate of the oscilloscope that was used to record the voltage signal $U_{\text {Oszi }}(t)$, being 0.1 ns. By that it follows that the error introduced by this approach is of no consequence to the interpretation of the experimental data even if - as in the following $-\varepsilon$ is regarded to equal 0 . The surface temperature after $t_{\mathrm{n}}$ is given by $T\left(t_{\mathrm{n}}\right)=T_{\mathrm{RT}}+\Delta T\left(t_{\mathrm{n}}\right)$. The iterative determination of $\Delta T\left(t_{\mathrm{n}}\right)$ allows to consider the temperature dependences of $\kappa$ and $k$ and yields to

$T\left(t_{n}\right)=T_{R T}+\frac{2 \cdot I_{\mathrm{abs}}\left(t_{\mathrm{n}-1}\right)}{\kappa\left(T_{R T}+\Delta T\left(t_{\mathrm{n}-1}\right)\right)} \sqrt{\frac{k\left(T_{R T}+\Delta T\left(t_{\mathrm{n}-1}\right)\right)}{\pi} \cdot t_{\mathrm{n}}}$.

For the following calculations, the values of $\kappa(T)$ and $k(T)$ are taken from [18].

Section (1) of the curve shown in Fig. 3 can be described by

$R_{\mathrm{Si}}\left(t_{\mathrm{n}}\right)=C \cdot t_{\mathrm{n}}+D$

where $C$ and $D$ result from regression analyses of the timeresolved reflection signals (compare right ordinate in Fig 3). $C$ and $D$ need to be determined for each $U_{\text {Oszi }}(t)$ measurement individually. When equation (7) is solved for $t_{n}$ and then inserted into equation (8), it follows that

$R_{S i}\left(T\left(t_{\mathrm{n}}\right)\right)=C \cdot \frac{\kappa^{2}\left(T^{\prime}\right) \cdot \pi}{4 \cdot k\left(T^{\prime}\right) \cdot I_{\mathrm{abs}}^{2}\left(t_{\mathrm{n}-1}\right)} \cdot \Delta T\left(t_{\mathrm{n}}\right)^{2}+D$

scales for temperatures below $T_{\mathrm{m}}$ with $\Delta T^{2}$ or $T\left(t_{n}\right)^{2}$, respectively, where $T^{\prime}=T_{\mathrm{RT}}+\Delta T\left(t_{\mathrm{n}-1}\right)$.

According to equation (9) one would expect a quadratic dependence of $R_{\mathrm{Si}}(T)$ on $T$, if the material properties were constant. However, the increase of $R_{\mathrm{Si}}(T)$ is dominated by the quotient $\kappa^{2}(T) / k\left(T^{\prime}\right)$, leading to an almost linear behavior of $R_{\mathrm{Si}}(T)$ for $T<T_{\mathrm{m}}$.

The dotted-dashed line in Fig. 5 shows the temperature dependence of the reflectivity of crystalline silicon in the solid phase for the entire temperature interval between $T_{\mathrm{RT}}$ and $T_{\mathrm{m}}$ as determined by the method described above.

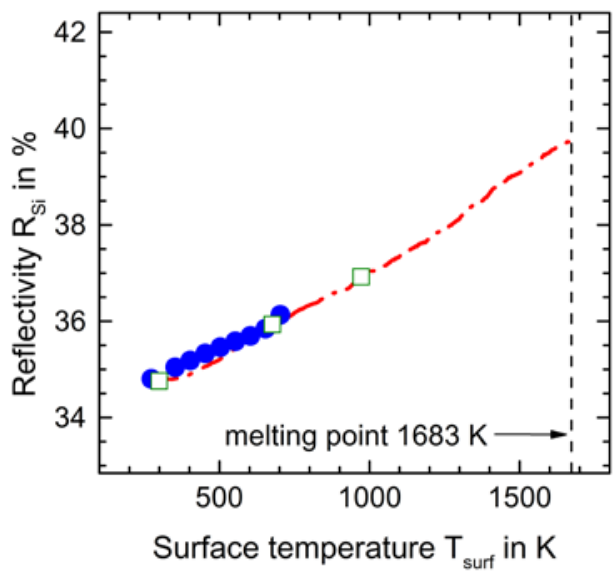

Fig. 5 Reflectivity of crystalline silicon as a function of surface temperature for the probe wavelength $\lambda=632.8 \mathrm{~nm}$ in the entire temperature interval between $T_{\mathrm{RT}}$ and $T_{\mathrm{m}}$ according to equation

(9). (O) denote experimental values of $R_{\mathrm{Si}}(T)$ published in [9] and [5] (口), respectively.
For comparison, we have included published values determined by means of ellipsometry measurements [5, 9]. Note that in the cited ellipsometry experiments, precautions have been taken to reduce oxidation at elevated temperatures (e. g. heating and measurement procedure under an inert gas atmosphere). Therefore, the discrete data points can be assumed to represent the exact reflectivity values for the respective temperatures and are suitable to validate the elaborated approach. Even though the relation between reflectivity and temperature was not directly measured but only deducted from recorded voltage signal, the presented approach describes the temperature dependence of the reflectivity of crystalline silicon between room temperature and the melting point in excellent agreement with experimental data. The $R_{\mathrm{Si}}(T)$-curve in Fig. 5 can be approximated in the interval $T_{\mathrm{RT}}<T<T_{\mathrm{m}}$ by

$R_{\mathrm{Si}}(T)=6.409 \times 10^{-9} \cdot T^{2}+2.502 \times 10^{-5} \cdot T+0.339$

with a coefficient of determination of $99.88 \%$. When inserting equation (9) into equation (1), the expression can be solved for the laser-induced temperature increase

$\Delta T\left(t_{\mathrm{n}}\right)=\frac{2 \cdot I_{\mathrm{abs}}\left(t_{\mathrm{n}-1}\right)}{\kappa\left(T^{\prime}\right)} \cdot \sqrt{\frac{k\left(T^{\prime}\right)}{\pi \cdot C}} \cdot \sqrt{\frac{U_{O S z i}\left(t_{\mathrm{n}}\right)-B}{A \cdot I_{\mathrm{HeNe}}}-D}$,

which can thus be determined as a direct result of the recorded photodiode voltage signals $U_{\text {Oszi }}\left(t_{n}\right)$.

The temporal evolution of the laser-induced temperature increase of crystalline silicon is calculated according to equation (11) and is shown for different pulse durations in Fig. 6. The laser intensity was adjusted for each pulse duration in the way that the laser-silicon interaction area reaches the melting temperature.

After the end of the laser pulse, the laser-silicon interaction area cools down from $T_{\mathrm{m}}$ to $T_{\mathrm{RT}}$ within several microseconds. We have shown earlier that a repeated irradiation of the same area (e.g. irradiation with high pulse overlap) leads to heat accumulation in the substrate and to a reduction of the laser fluence needed to melt the surface [19].

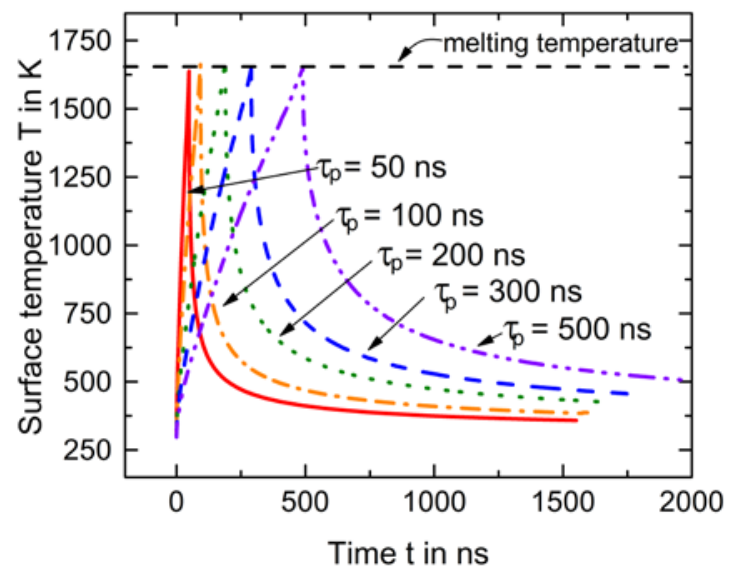

Fig. 6 Time-resolved evolution of the temperature increase during laser irradiation with different pulse durations as calculated from the detected voltage signals with the help of equation (11). 
The maximum cooling rates of the hot solid $d T / d t$ can be extracted from Fig. 6 and are shown in Fig. 7 as a function of pulse duration. They occur directly after the end of the laser pulse. For laser fluences equivalent to the melt threshold it applies that the shorter the laser pulse duration, the higher the maximum cooling rate. This is indicated by the fit to the measured data (dashed line), which gives

$$
d T / d t \sim 1 / \sqrt{\tau_{p}} .
$$

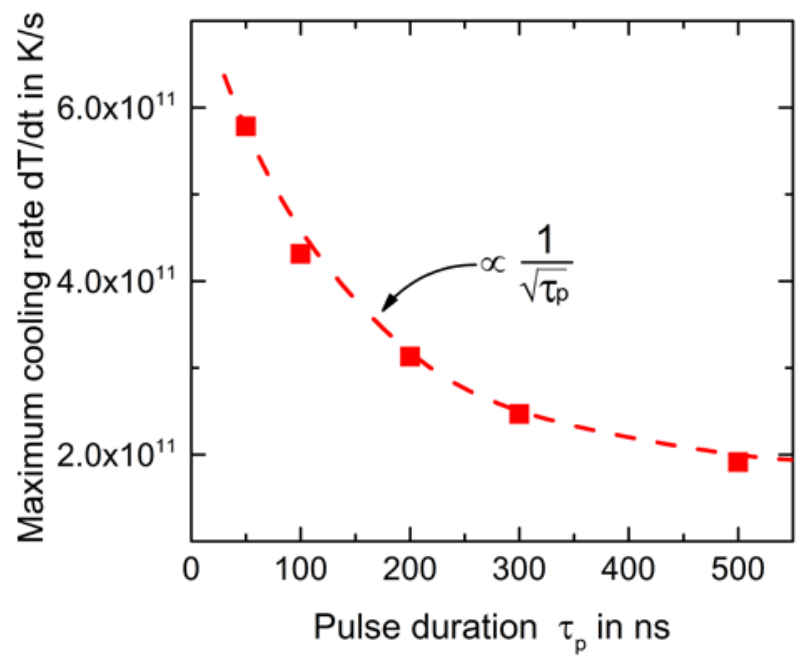

Fig. 7 Maximum cooling rates as a function of pulse duration. The dashed line corresponds to the interpolation of the measured data.

For the pulse durations investigated in this paper, the maximum cooling rates of the hot silicon lie in the order of magnitude of $10^{11} \mathrm{~K} / \mathrm{s}$. These cooling rates are three orders of magnitude below the cooling rates that lead to amorphous crystallization [20]. Thus, crystalline regrowth is ensured for laser-induced melting processes with pulse durations in the nanosecond regime.

\section{Summary}

The temperature-dependence of the reflectivity of crystalline silicon $R_{\mathrm{Si}}(T)$ was determined in the entire temperature range between room temperature and the melting point by means of time-resolved reflectivity measurements during pulsed laser irradiation. This approach as well as the experiments presented in this paper extend the analyzed temperature range in a consistent and comprehensive manner. Before this work, the reflectivity values of crystalline silicon were only reported for discrete temperatures (e.g. in [3-13, 21-24]).

The reflectivity of silicon at $T_{\mathrm{m}}$ was determined to be $39.8 \% \pm 0.6 \%$ for $\lambda=632.8 \mathrm{~nm}$. The elaborated methodology to convert the measured voltage signals into reflectivity or temperature values respectively suggests a very simple technique for the experimental, in-situ temperature determination in the laser-material interaction area with subnanosecond time resolution. What is more, the time scale of heating, measuring and cooling is too short to allow for significant oxidation of the silicon surface that might distort the measurement results $[13,14]$. For the first time, this approach was successfully applied to continuously monitor the entire heating and cooling phase of the silicon surface during single pulse irradiation with laser pulse durations between 50 ns and 500 ns. Cooling rates of the hot silicon in the order of $10^{11} \mathrm{~K} / \mathrm{s}$ that still allow for crystalline regrowth were found. It followed that laser-induced melting processes with pulse durations in the nanosecond regime ensure crystalline regrowth.

\section{References}

[1] K. Phillips, H. Gandhi, E. Mazur and S. Sundaram: Adv. Opt. Photonics 7, (2015) 4.

[2] M. Gower: Proc. SPIE 4559, "MEMS Components and Applications for Industry, Automobiles, Aerospace, and Communication” ed. by H. Helvajian, S. Janson, F. Lärmer, (2001) p. 53.

[3] M. Green: Solar Energy Materials and Solar Cells, 92, (2008) 11.

[4] G. Jellison Jr.: "Semiconductors and Semimetals" ed. by R. Wood, C. White and R. Young, (Elsevier, 1984) p. 95-164.

[5] G. Jellison Jr. and F. Modine: Appl. Phys. Lett., 41, (1982) 2.

[6] G. Jellison Jr. and H. Burke: J. Appl. Phys., 60, (1986) 2.

[7] J. Heller and J. Bartha: Proc. Conference on Lasers and Electro-Optics, Baltimore (1999) p.450.

[8] H. Li: J. Phys. Chem. Ref. Data, 9, (1980) 3.

[9] G. Vuye, S. Fisson, V. Van, Y. Wang, J. Rivory and F. Abelès: Thin Solid Films, 233, (1993) 1.

[10] G. Jellison: Opt. Mat., 1, (1992) 1.

[11]G. Ivlev and E. Gatskevich: Semiconductors, 34, (2000) 7.

[12] J. Boneberg, O. Yavas, B. Mierswa and P. Leiderer: Phys. Status Solidi B, 174, (1992) 1.

[13]N. Chaoui, J. Siegel, J. Solis and C. Afonso: J. Appl. Phys., 89, (2001) 7.

[14]G. Jellison Jr. and D. Lowndes: Appl. Phys. Lett., 51, (1987) 5.

[15]A. Al-Bayati, K. Orrman-Rossiter, J. van den Berg and D. Armour: Surf. Sci., 241, (1991) 1.

[16]M. von Allmen and A. Blatter: "Laser-Beam Interactions with Materials: Physical Principles and Applications” ed. by U. Gonser, (Springer, 1995) p. 43ff.

[17]E. Gatskevich, P. Prikryl and G. Ivlev: Math. Comput. Simul., 76, (2007) 1-3.

[18]A. Goldsmith, T. Waterman and H. Hirschhorn: "Handbook of Thermophysical Properties of Solid Materials: Elements “ (Macmillan, New York 1961)

[19]M. Diez, W. Zhu, M. Ametowobla and A. Letsch: Lecture on „Influencing factors on the laser doping (LD) process for solar cells" during "Stuttgarter Lasertage”, Stuttgart, (14.06.2012).

[20]R. Tsu, R. Hodgson, T. Tan and J. Baglin: Phys. Rev. Lett., 42, (1979) 20.

[21] M. Lampert, J. Koebel and P. Siffert: J. Appl. Phys., 52, (1981) 2.

[22] M. Toulemonde, S. Unamuno, R. Heddache, M. Lampert, M. Hageali and P. Siffert: Appl. Phys. A, 36, (1985) 1.

[23]W. Spitzer and H. Fan: Phys. Rev., 106, (1957).

[24]H. Weakliem and D. Redfield: J. Appl. Phys., 50, (1979) 3.

(Received: August 29, 2017, Accepted: October 17, 2017) 